\title{
Deux Dinopsyllus nouveaux du Zaïre (Siphonaptera, Hystrichopsyllidae)
}

\author{
par J.-C. BEAUCOURNU et U. RAHM \\ Section d'Epidémiologie du Laboratoire de Parasitologie (U.E.R. Santé dans la Collectivité), \\ Faculté de Médecine, F 35000 Rennes, \\ et Muséum d'Histoire naturelle. 4051 Bâle, Suisse.
}

\section{Résumé.}

Description de Dinopsyllus (D.) traubi n. sp. parasite de Graphiurus (Claviglis) murinus vulcanicus Lönnberg et Gyldenstolpe et de D. (D.) macrochaetus n. sp. parasite de divers Muridae.

\section{Summary:}

Two new Dinopsyllus (Siphonaptera, Hystrichopsyllidae) from Zaïre.

Description of Dinopsyllus (D.) traubi n. sp. parasitic on Graphiurus (Claviglis) murinus vulcanicus Lönnberg and Gyldenstolpe and of $D$. (D.) macrochaetus n. $\mathrm{sp}$. parasitic on several Muridae.

Dans un abondant matériel de siphonaptères récolté par l'un de nous (U.R.) (1) dans la région du lac Kivu, République du Zaïre, nous avons identifié sept espèces appartenant au genre Dinopsyllus. Nous n'avons pas retrouvé D. wansoni Berteaux 1947, cependant décrit de cette région, mais, par contre, nous avons relevé la présence de deux espèces que nous estimons nouvelles et que nous décrivons ci-après. La liste complète des puces présentes dans nos prélèvements sera donnée dans une note ultérieure.

(1) Subside de la Life Sciences Division, Army Research Office, Grant n DAHC 19-68-G-0006. Annales de Parasitologie humaine et comparée (Paris), t. 50, $\mathrm{n}^{\circ} 1$ 


\section{Dinopsyllus (Dinopsyllus) traubi n. sp.}

\section{Matériel de description.}

Le mâle holotype récolté sur Graphiurus (Claviglis) murinus vulcanicus Lönnberg et Gyldenstolpe, 1925, Lemera (Zaïre) $\left(28^{\circ} 47^{\prime} \mathrm{E}, 2^{\circ} 10^{\prime} \mathrm{S}\right)$, altitude $2000 \mathrm{~m}, 24-6-65$.
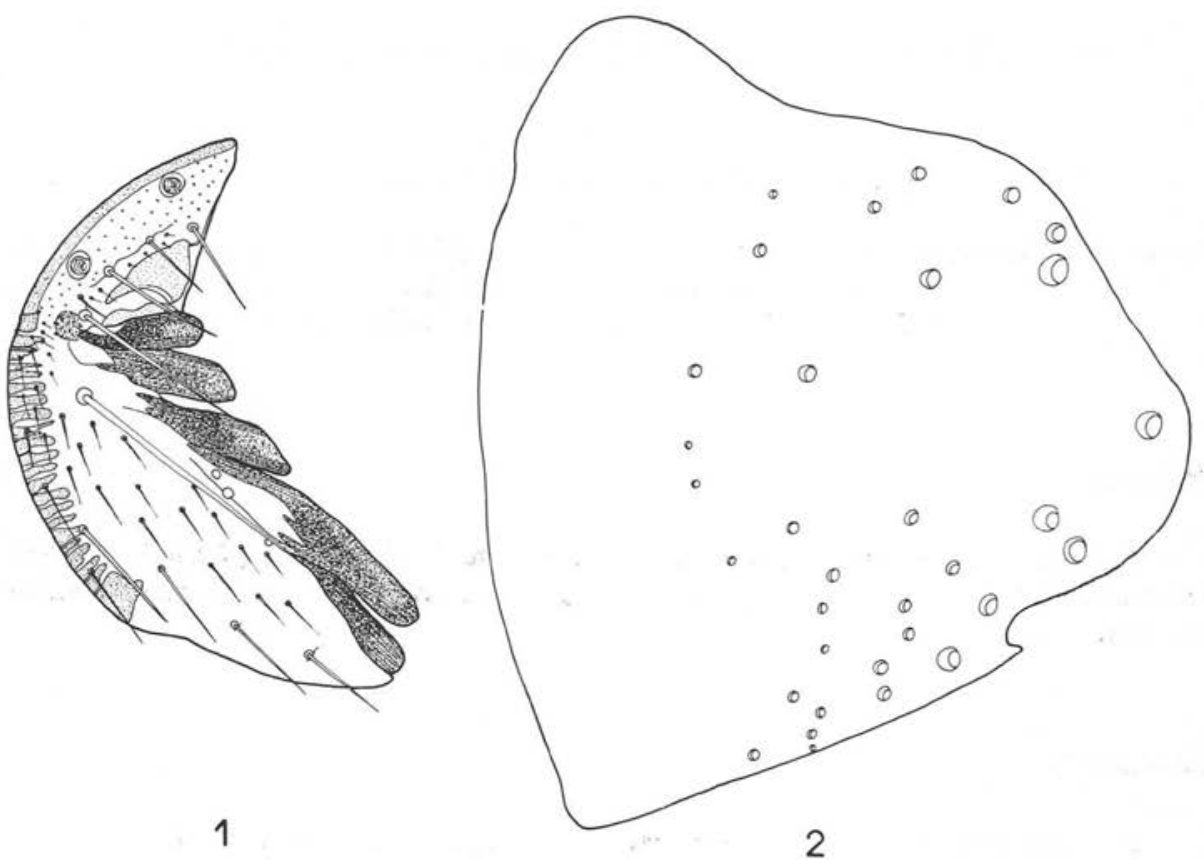

Fig. 1. - Dinopsyllus traubi n. sp., holotype (Lemera): partie antérieure de la capsule céphalique.

FIG. 2. - Dinopsyllus traubi n. sp., holotype (Lemera): sternite VIII.

Cet exemplaire est déposé dans les collections du premier signataire.

Nous sommes heureux de dédier cette nouvelle espèce au $\mathrm{P}^{\mathrm{r}}$ Traub en hommage à ses travaux sur les Siphonaptères.

\section{Description.}

Capsule céphalique (fig. 1) arrondie et courte, tubercule frontal un peu au-dessus du milieu du bord antérieur. Epaississement de la marge frontale (en-dessous du tubercule) large, sans variation notable de largeur d'une extrémité à l'autre. Palpe labial long, dépassant les trois quarts de la coxa I. 
Pronotum portant deux rangées de soies. Cténidie de 29 dents, la longueur de celles-ci ( $125 \mu$ sur la ligne médio-dorsale) étant égale aux deux tiers du pronotum $(189 \mu)$.

Pattes: 14 soies sur la face externe du tibia III.

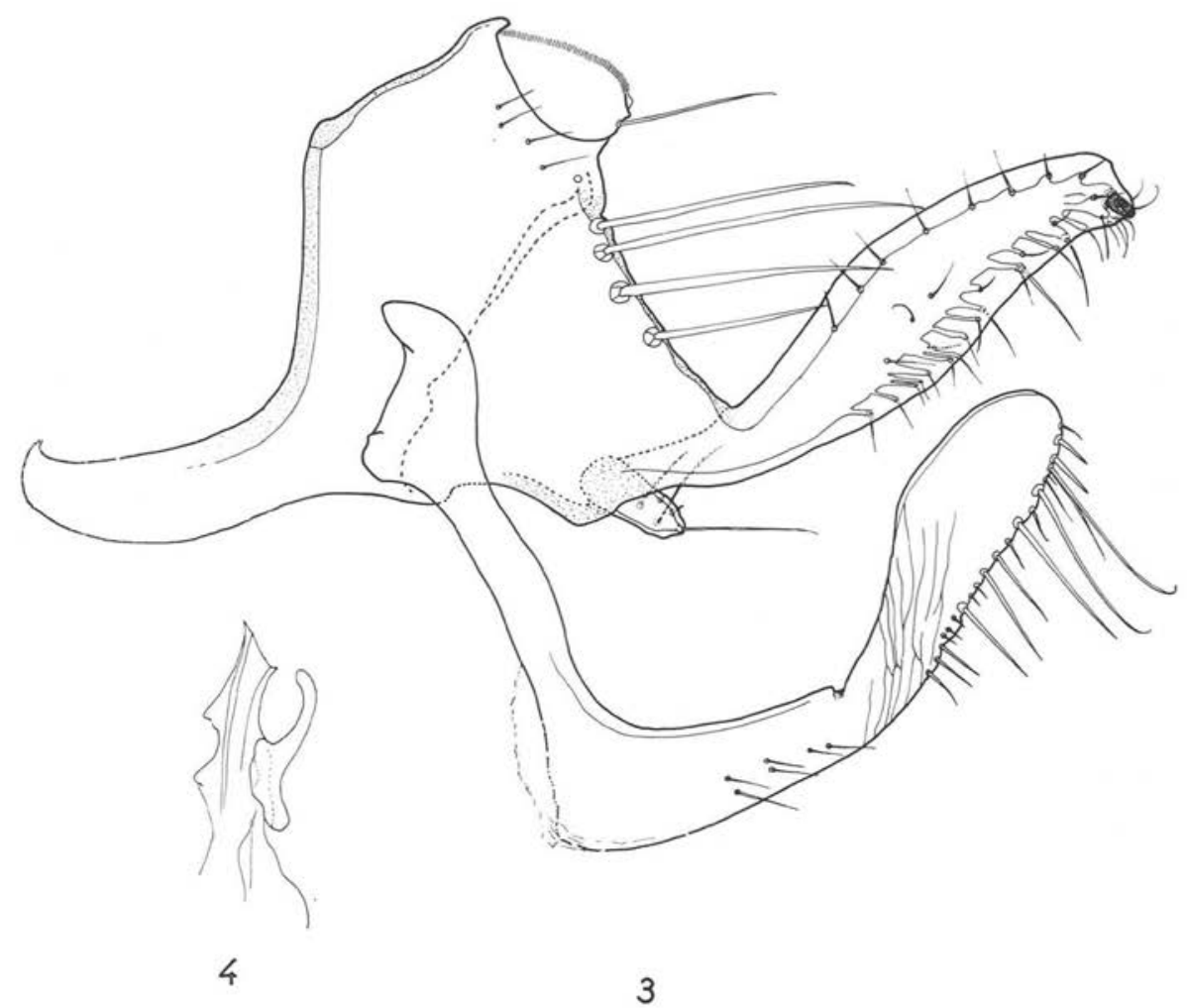

FIG. 3. - Dinopsyllus traubi n. sp., holotype (Lemera): segment IX.

FIg. 4. - Dinopsyllus traubi n. sp., holotype (Lemera): extrémité du ductus ejaculatorius et hamulus.

Abdomen: tergite I portant une courte épine d'un côté seulement ; tergites II à $\mathrm{V}$ portant respectivement 7 et 7,8 et 8,9 et 11,7 et 7 épines de chaque côté.

Segments modifiés: sternite VIII (fig. 2) arrondi à son extrémité laquelle porte une seule soie ; une encoche nette à la partie inféro-apicale de ce sternite, suivie par une très fine denticulation de la marge, immédiatement au-dessus.

Basimère (fig. 3) sans caractère particulier; manubrium non élargi à son apex. Télomère élargi en son milieu, de forme générale en $\mathrm{S}$; chétotaxie de son 
bord dorsal commençant au tiers inférieur et faite de soies relativement longues, dépassant nettement la marge de leur bord d'implantation. Processus mobile de la base du télomère portant les soies habituelles mais celle de l'apex très longue.

Sternite IX: légèrement élargi à l'apex; sétation marginale sans intervalle libre, les deux longues soies supérieures recourbées. Striation cuticulaire, immédiatement au-dessus de la «pseudarthrose » du bras distal, très accentuée.

Hamulus («Crochet du phallosome») (fig. 4) long et recourbé, voisin de celui de $D$. longifrons.

Taille: mâle (en préparation microscopique) : $2,50 \mathrm{~mm}$.

Femelle inconnue.

Discussion: D. traubi vient se placer dans le groupe des deux espèces affines D. kempi Jordan et Rothschild, 1913 et D. pringlei Hubbard, 1963. Il se sépare immédiatement de la première par la forme du sternite VIII. D. pringlei, très proche, s'en distingue au moins par le nombre d'épines résiduelles abdominales, la forme et la sétation du télomère et du sternite VIII.

Il est à noter que les rares exemplaires connus de $D$. kempi proviennent de Graphiurus murinus (Muscardinidae), ainsi que l'holotype de D. pringlei. D. traubi, provenant de ce même rongeur, souligne la cohérence écologique et phylétique vraisemblable du « groupe kempi».

\section{Dinopsyllus (Dinopsyllus) macrocbaetus n. sp.}

\section{Matériel de description.}

Mâle holotype récolté sur Malacomys longipes centralis de Winton, Lemera (Zaïre), $2000 \mathrm{~m}, 21-10-62$; un mâle paratype même hôte, Lemera, 25-5-67 ; un mâle paratype, même hôte, Bogamanda (Zaïre), 2200 m, 22-9-69; un mâle paratype, sur Thamnomys venustus kempi Dollman, 1911. Lemera, 11-66; un mâle paratype sur Hybomys univittatus (Peter), Lemera, 27-5-67.

L'holotype et un paratype sont déposés dans les collections de l'U.S. National Museum. Deux paratypes sont dans les collections du premier signataire, un paratype dans celles du Muséum d'Histoire naturelle de Bâle.

Nous remercions très vivement le $\mathrm{P}^{r} \mathrm{R}$. Traub d'avoir bien voulu nous confier l'exemplaire de D. macrochaetus en sa possession et de nous en avoir autorisé la description.

\section{Description.}

Capsule céphalique (fig. 5) courte et arrondie; tubercule frontal relativement développé situé à la moitié de la marge antérieure céphalique. Epaississement de la marge frontale (en-dessous du tubercule) large, ne s'amenuisant pas aux extrémités. Palpe labial atteignant environ les deux tiers de la Coxa $I$. 
Pronotum portant deux rangées de soies. Chez l'holotype et un paratype, une soie ou deux soies sont déplacées en avant de la rangée antérieure et pourraient figurer une troisième rangée vestigiale.
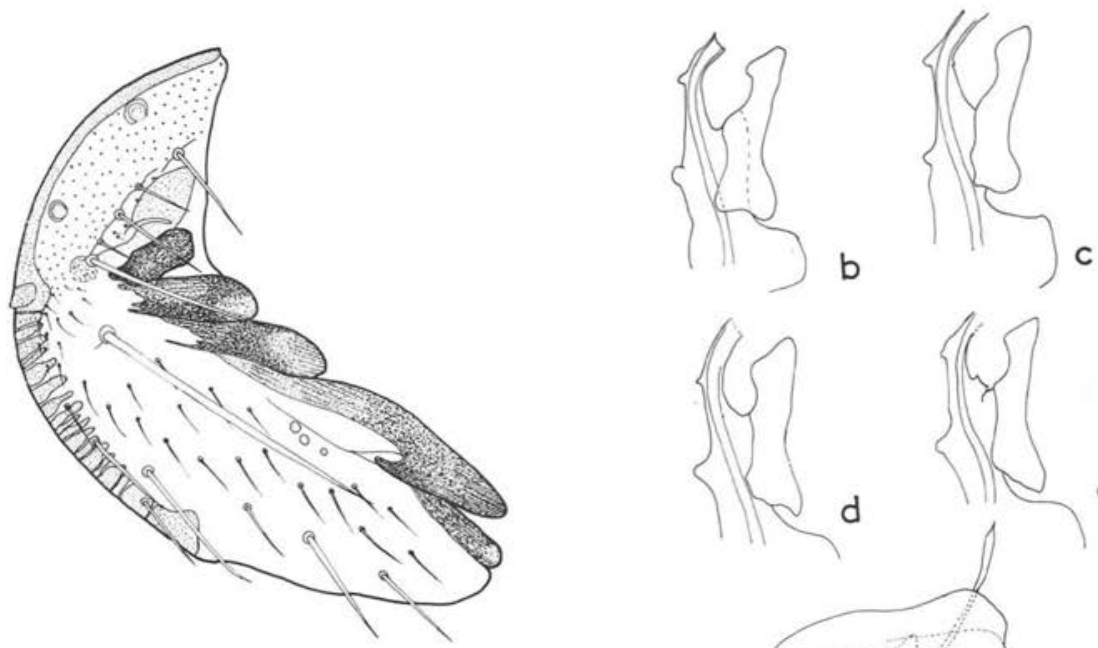

5

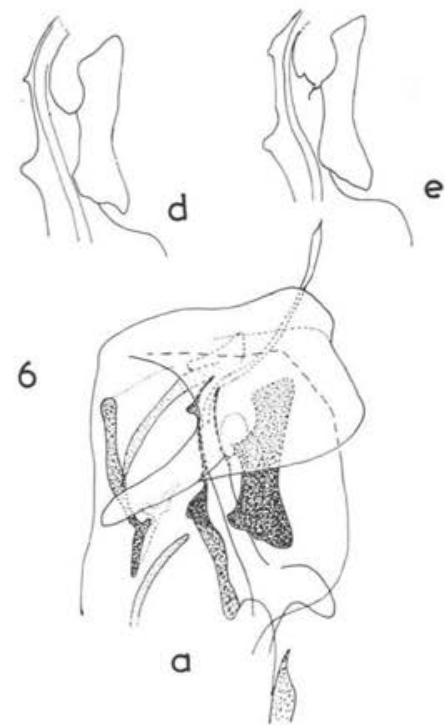

Fig. 5. - Dinopsyllus macrochaetus n.sp., paratype (Lemera): partie antérieure de la capsule céphalique.

FIg. 6. - Dinopsyllus macrochaetus n. sp.; extrémité du ductus ejaculatorius et hamulus; a: holotype ; b, c, d, e: paratypes.

Dents de la cténidie pronotale à peu près égale à la moitié du pronotum (holotype : $108 \mu$ et $228 \mu$ ) mesuré sur la ligne médiodorsale. Cténidie de 37 dents chez l'holotype et trois paratypes, $38 \mathrm{chez}$ un paratype.

Pattes: soies des pattes relativement fortes. De 14 à 18 soies sur la face externe du tibia III.

Abdomen: soies abdominales de longueur normale. Pas de cténidie vestigiale sur le tergite I. Sur les tergites II à V (tergite VI sans cténidie), le nombre d'épines est le suivant : 
Holotype Paratype 1 Paratype 2 Paratype 3 Paratype 4

\begin{tabular}{|c|c|c|c|c|c|c|}
\hline T. II & . & $4-2$ & $4-5$ & $4-5$ & $5-4$ & $5-4$ \\
\hline T. III & .. & $5-4$ & $6-5$ & $5-5$ & $5-6$ & $6-5$ \\
\hline T. IV & . & $7-6$ & $8-7$ & $10-10$ & $8-7$ & $6-8$ \\
\hline T. V & & $3-3$ & $1-1$ & $2-2$ & $1-1$ & $2-1$ \\
\hline
\end{tabular}

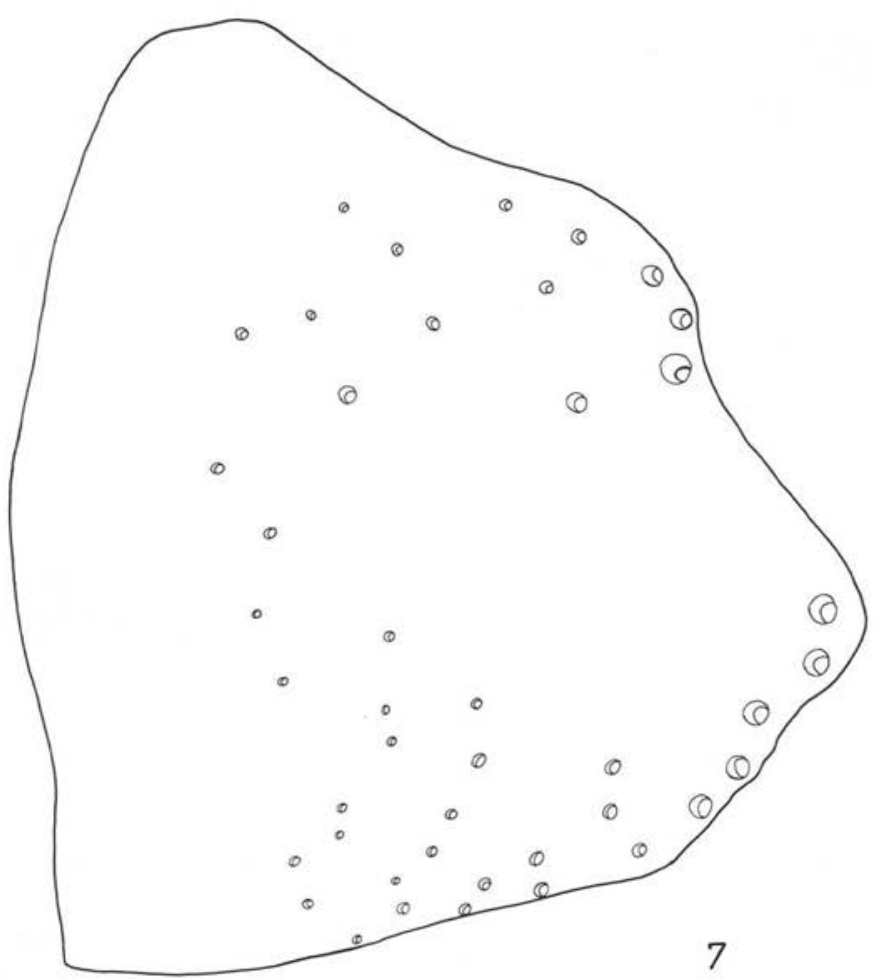

FIG. 7. - Dinopsyllus macrochaetus n. sp., paratype (Lemera): sternite VIII.

Segments modifiés: sternite VIII (fig. 7) à apex émoussé triangulaire, le bord postéro-dorsal étant rectiligne ou quelque peu concave; deux soies à l'apex; pas d'échancrure de la marge inférieure.

Basimère (fig. 8 ) non distinctif, le seul caractère notable étant la brièveté et le caractère massif du manubrium. Télomère très élargi, la partie la plus large se situant entre le milieu et les deux tiers de cette pièce. Sétation variable d'un exemplaire à l'autre. 


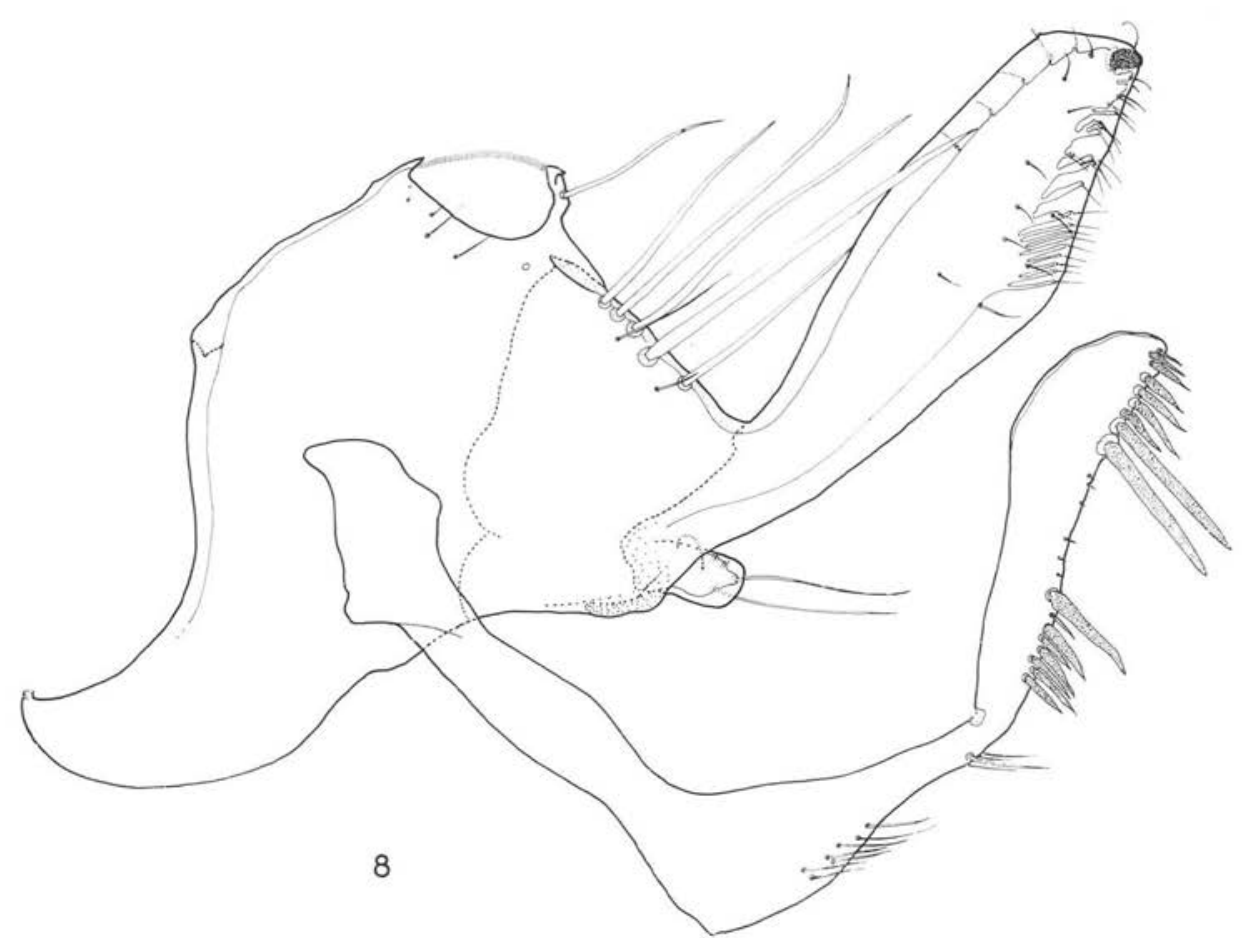

FIG. 8. - Dinopsyllus macrochaetus n. sp., paratype (Lemera) : segment IX.

Sternite IX : deux touffes, ou plaques, de soies sur la partie basale du bras distal. Soies de la portion apicale spiniformes, noires, séparées en deux zones par un très large intervalle; chez l'holotype cet espace est coupé en deux par une forte soie plus ou moins médiane.

Hamulus (fig. 6) : large et spatulé.

Taille: mâles (en préparation microscopique) : 2,9 à $3,5 \mathrm{~mm}$.

Femelle inconnue.

\section{Discussion :}

D. macrochaetus n. sp. se distingue des autres espèces connues par son sternite IX et la morphologie du crochet du phallosome: il ne peut en particulier être confondu avec aucun Dinopsyllus à crochet large (ellobius, zuluensis, abaris). 